\title{
Potential Use of Patient-Specific Induced Pluripotent Stem Cell for Liver Fibrosis Thalassemia Treatment Management
}

\author{
Susi Susanah ${ }^{1} \mathbb{D}$, Wahyu Widowati ${ }^{2 *}$, Nur Melani Sari ${ }^{1,3} \mathbb{D}$, Revika Revika ${ }^{4}$, Hanna Kusuma ${ }^{5}$, Rizal Rizal ${ }^{5,6} \mathbb{D}$, \\ Ahmad Faried ${ }^{3}$ iD \\ ${ }^{1}$ Department of Child Health, Faculty of Medicine, Universitas Padjadjaran, Dr. Hasan Sadikin Hospital, Bandung, Indonesia; \\ ${ }^{2}$ Department of Pharmacology, Faculty of Medicine, Maranatha Christian University, Bandung, Indonesia; ${ }^{3}$ Oncology and Stem \\ Cell Working Group, Universitas Padjadjaran, Dr. Hasan Sadikin Hospital, Bandung, Indonesia; ${ }^{4}$ Department of Biotechnology, \\ Faculty of Technobiology, Atma Jaya Catholic University of Indonesia, South Tangerang, Banten, Indonesia; ${ }^{5}$ Biomolecular and \\ Biomedical Research Center, Aretha Medika Utama, Bandung, Indonesia; ${ }^{6}$ Department of Electrical Engineering, Biomedical \\ Engineering, Faculty of Engineering, Universitas Indonesia, Depok, Indonesia
}

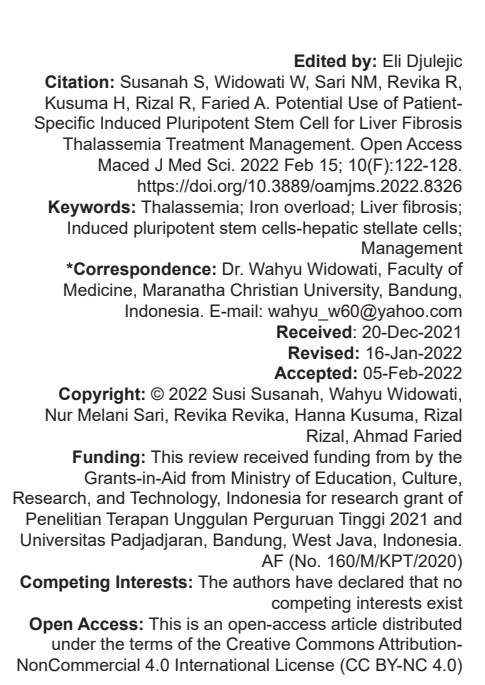

\section{Abstract}

Thalassemia is the most common inherited single gene blood disease worldwide and present a significant health problem in the world. Approximately, 1.5\% of the global populations (An estimated 80-90 million people) are carriers of $\beta$-thalassemia. Around $5 \%$ of Indonesia population is thought to carry the thalassemia gene. The globin imbalance in $\beta$-thalassemia major causes hemolysis and ineffective erythropoiesis which results in anemia leading to increases of iron absorption. Furthermore, repeated blood transfusion and long-term increased iron absorption will lead to excessive accumulation of iron in vital organs, especially in the liver, causes liver fibrosis then leading to liver disease. Iron overload can be controlled by iron chelating drugs with the risk of side effects; therefore, a breakthrough is needed. Stem cell technology has a potential to provide novel insight in thalassemia major, through induced pluripotent stem cells (iPSCs) who has the ability to differentiate into hepatic stellate cells (HSCs)-like cells. iPSCs derived HSC-like cells (iPSC-HSCs) present the phenotypic and functional characteristics of HSCs. The utilization of iPSCs is a new option in personalized thalassemia management.

\section{Introduction}

Thalassemia is the most common form of inherited anemia worldwide and is associated with significant morbidity and mortality rates leading to a significant global health problem. Globally, around $7 \%$ of pregnant women carry $\beta$ or $\alpha$ thalassemia, or hemoglobin S, C, D Punjab, or E, and over $1 \%$ of couples are at risk of having children with a thalassemia disease [1]. It is reported around $5 \%$ of individual Indonesian were carrying thalassemia and predicted at least 2,500 children with thalassemia major were born every year [2].

The term "thalassemia" refers to a group of autosomal recessive blood diseases characterized by the decreased or absent synthesis of normal globin chains. From a clinical point of view, the most relevant types are $\alpha$ and $\beta$-thalassemia, resulting from the decreased synthesis of one of the two types of polypeptide chains ( $\alpha$ or $\beta$ ) that form the normal adult human hemoglobin $(\mathrm{Hb})(\alpha 2 \beta 2)$ and reduced hemoglobinization. The basic defect in $\beta$-thalassemia is reduced or absent production of $\beta$ globin chains with a relative excess of $\alpha$ chains. The $\alpha$ globin chains accumulate and precipitate in the erythroid precursors forming inclusion bodies that cause oxidative membrane damage and ineffective erythropoiesis lead hemolytic anemia [3]. Individuals with $\beta$-thalassemia major have severe anemia and hepatosplenomegaly; they usually come to medical attention within the first 2 years of life. Without treatment, affected children have severe failure to thrive and shortened life expectancy [4].

Classically, the spectrum of thalassemia is divided into thalassemia major, intermedia, and minor (trait); based on a need for transfusion, practically it is categorized into two groups: thalassemia dependent transfusion (TDT) 


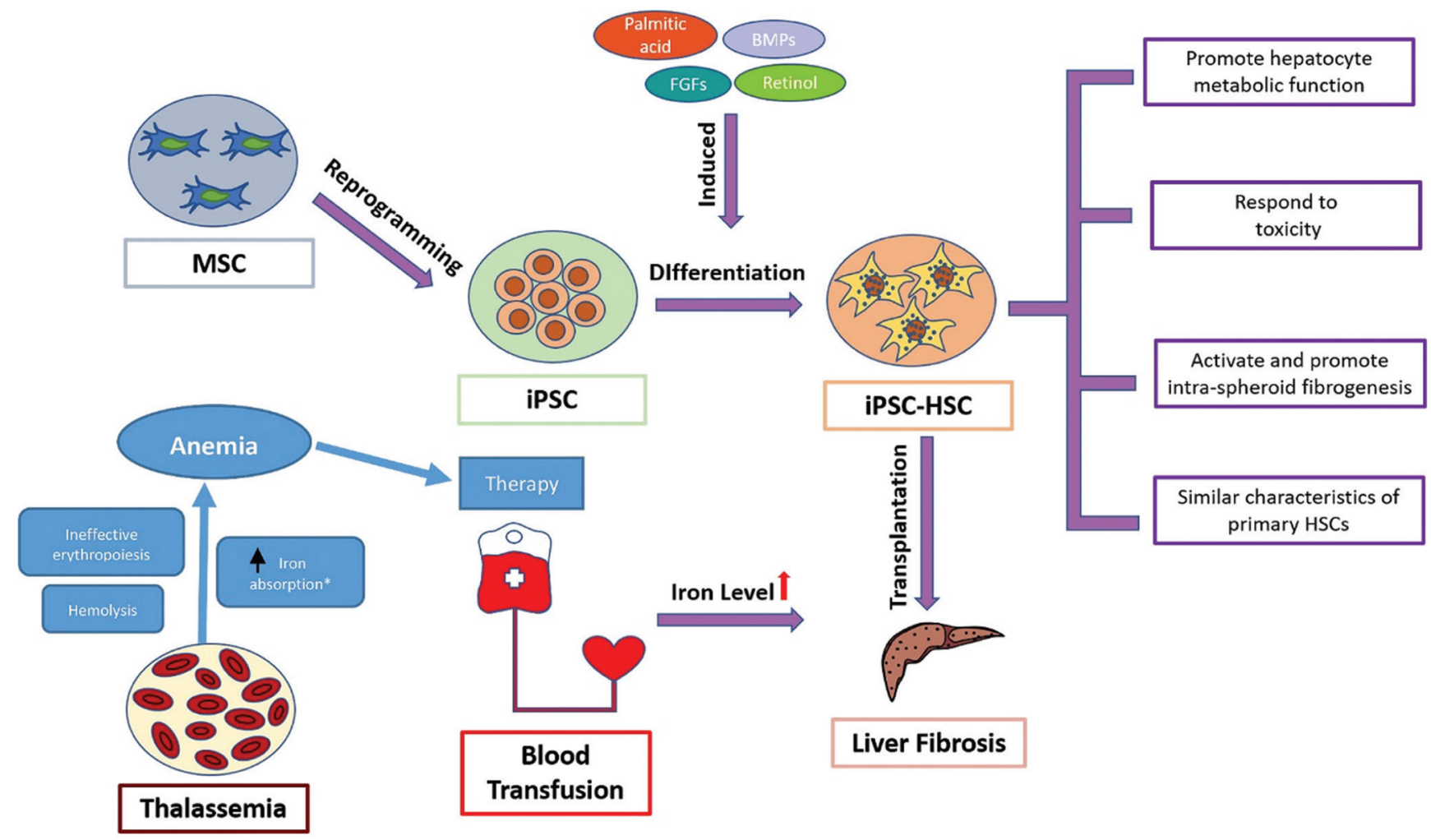

Figure 1: Induced pluripotent stem cells (iPSCs)- hepatic stellate cells (HSC) pathway for liver fibrosis to thalassemia therapy. Blood transfusion is a primary therapy for thalassemia, but that therapy can cause iron accumulation lead to liver fibrosis. Cell-based therapy is an alternative therapy for liver fibrosis, such as iPSCs. iPSCs can be generated from mesenchymal stem cells by reprogramming. Induced palmitic acid, bone morphogenetic proteins, fibroblast growth factors, and retinol can differentiate iPSC to iPSC-HSC. HSCs are the key to wound healing a liver fibrosis. iPSC-HSC have characteristics of primary HSCs, activate and promote intra-spheroid fibrogenesis, respond to hepatocyte-mediated toxicity, and promote hepatocyte metabolic function. iPSC-HSC transplanted to thalassemia patient with liver fibrosis, so the patient could continue to receive blood transfusion

equal to thalassemia major and non-transfusion dependent thalassemia [4]. People with thalassemia major will require a regular blood transfusion to treat their anemia. However, one major drawback of blood transfusion is iron overload. Excessive levels of iron due to blood transfusion accompanied by increased iron absorption cause accumulation of iron in the organs, especially the liver [5]. The thalassemia-blood transfusion was shown in Figure 1.

Hepatocytes are the main site of iron $(\mathrm{Fe})$ storage and also the principal site of synthesis of hepcidin, a hormone responsible for the regulation of iron transport to the extracellular space. In cases of thalassemia, the excess iron initially accumulates in macrophages and later in hepatocytes, while hypoxia caused by dyserythropoietic intensifies erythropoietin production leading to further suppression of hepcidin production and increased transferrin bound iron (TBI) and non-TBI (NTBI) plasma levels [6].

Excessive levels of iron in the bloodstream exceed the capacity of plasma transferrin to bind iron and lead to the appearance of NTBI in the plasma which consists of a highly toxic iron species. The liver rapidly clears NTBI from the serum through divalent metal transporter 1 and incorporates it into ferritin. However, once the protective storage efficiency of ferritin is exceeded, unbound iron accumulates in the hepatocytes and leads to severe oxidative stress and overproduction of toxic reactive oxygen species, which can oxidize lipid membrane, protein, and organelle membrane, leading to cell damage and death [5]. The subsequent hepatic inflammation and necrosis lead to fibrosis and cirrhosis [7]. The development of fibrosis in the absence of inflammation has also been found to be associated with iron accumulation in the liver and relates to the direct activation of stellate cells [8].

To date, iron overload is treated by iron chelation drugs, such as deferoxamine, deferiprone, and deferasirox. Besides its efficacy, as a drug that is consumed for a long time, there is a risk of side effects. However, iron chelator therapy cannot completely eliminate the risk of iron accumulation in the liver leading to liver fibrosis. Hepatitis virus infection related to blood transfusion is another cause of liver fibrosis in TDT patients. Elalfy et al. reported liver fibrosis even in young Egyptian $\beta$-thalassemia major patients [9].

Cell-based therapy, a technique to modify a patient's cells or cells from the donor, can be used to treat various diseases. The primary function of cell-based therapy is to fight disease safely and effectively [10]. Stem cell-based therapy can migrate to the host site and differentiation with specific characters. The classification of stem cell based on differentiation 
is totipotent, pluripotent, multipotent, oligopotent, and unipotent [11]. Mesenchymal stem cells (MSCs) are a commonly used cell-based therapy, but the plasticity of MSCs is rapidly lost during expansion [12].

Stem cells especially induced pluripotent stem cells (iPSCs) can be an alternative treatment for liver fibrosis. The iPSCs differentiate to hepatic stellate cells (HSCs)-like cells. HSCs is a key to wound healing liver fibrosis regarding injured liver condition, damaged hepatocytes, and immune cells will secrete a signal that will be received by HSCs [13]. Then, HSCs is transdifferentiated into activated myofibroblastlike cells. Activated HSCs increase the production of extracellular matrix (ECM), reducing degradation, and becoming the main fibrogenic cell type of the liver [14].

\section{Thalassemia Therapy}

Despite important advances in curative approaches such as stem cell transplantation and promising results of gene therapy, blood transfusions, and iron chelation remain as cornerstones of thalassemia management [4], [15]. Blood transfusions are needed to maintain the pre-transfusion $\mathrm{Hb}$ level $9.5-10.5 \mathrm{~g} / \mathrm{dL}$, to prevent growth impairment, bone marrow suppression activity, inhibition of increased gastrointestinal iron absorption, and prevent a progressive increase in spleen size [4]. While iron chelator drugs aim to reduce iron overload caused by repeated blood transfusions and increase iron absorption to maintain safe levels of body iron. Practically, an iron chelator is indicated to start after the first 10-20 transfusions, or when the ferritin level rises above $1000 \mu \mathrm{g} / \mathrm{L}$ [4]. The study by Susanah et al. found that even in newly diagnosed $\beta$-thalassemia major an increase in iron status has occurred due to erythropoiesis drive and iron overload might occur earlier [16].

A novel approach of therapy for thalassemia major is gene therapy. Several clinical trials are investigating the safety and efficacy of gene addition and gene editing to restore $\mathrm{Hb}$ synthesis in $\beta$-thalassemia. Most of the products are gene addition-based technologies, while few are gene-editing strategies that aim to reactivate fetal $\mathrm{Hb}$. However, this therapy needs conditioning myeloablative, which is associated with short and long toxicity in hematopoietic and non-hematopoietic tissues, to reduce the risk of graft failure. That toxicity effect lessens the willingness of patients to consider this therapy. Then, this therapy needs mobilization to mobilize hematopoietic stem and progenitor cells for transplantation. Before transplantation, this procedure needs an agent for mobilization such as hydroxyurea which can reduce the circulating CD34+, which is associated with myelosuppression. Furthermore, gene therapy and gene editing procedures are also known to be expensive [17].

\section{Stem Cells}

Stem cells are cells with the ability to selfrenew by dividing and differentiation with specific characteristics and functions under appropriate conditions and certain signals. Stem cells are classified as totipotent, pluripotent, multipotent, oligopotent, and unipotent [11]. Normally, stem cells can act as a replacement for lost cells due to injury, apoptosis, and physiological programmed turnover, a condition that is commonly found in the postnatal period. For therapy, stem cells can secrete soluble growth factors to repair injured tissues. The ideal stem cell is immunologically inert, obtained through sources easily accessible, as well as able to migrate into the host site, transfect, and express exogenous genes [12].

Based on traditional studies from somatic tissues, three common dogmas were found. First, it was thought that stem cells reservoirs only exist on tissues able to produce mature cells or regenerate under stressful conditions like intestinal epithelial and liver. Second, it has always been assumed that stem cells from a given somatic tissue have a differentiation potential that is limited to the mature cell populations that comprise the same tissue. Finally, the process of stem cell differentiation was thought to be unidirectional, thus the process was deemed irreversible [18].

All of these traditional dogmas, with current and modern scientific breakthroughs, were eventually debunked. First, stem cells reservoir can be found in the central nervous system that is identified in rodents and primates. Similarly, stem cells are found in the adult bone marrow. There are contain multipotent mesenchymal precursors. Second, stem cells are not limited to differentiating into adipocyte, chondrocyte, and hepatocyte. Recently, stem cells from muscle tissue can reconstitute hematopoietic cells in lethally irradiated mice. The other studies found hematopoietic stem cells from bone marrow-derived cells can reconstitute muscle fibers in a muscular dystrophy model [19]. Furthermore, differentiation stem cells are not limited to others germ layers. It is found that stem cells from bone marrow can generate neuronal-like cells in the brains of transplanted mice and can produce robust numbers of liver cells in a damaged liver. Finally, the process of stem cells differentiation may be quite flexible. Dedifferentiation process was shown in induced derived from the $\mathrm{C} 2 \mathrm{C} 12$ myoblast cell line [18].

\section{iPSCs}

iPSCs are a type of pluripotent stem cell. Pluripotent stem cells can differentiate into myocytes, hepatocytes, and neurons from three germ layers 
(endoderm, mesoderm, and ectoderm). iPSCs are a type of stem cell that is the most attractive for cell therapy because iPSCs can differentiate into all body cell types, rapid growth, high plasticity, and able to differentiate to multipotent-like cells. It was first discovered in 2006 when mouse fibroblasts are reprogrammed to be pluripotent and the result is similar to that observed in embryonic stem cells (ESCs) [20]. The reprogramming method used retroviral and transcription factors Oct4, Sox2, KIf4, and c-Myc. Retroviral vectors are used for iPSC transgene expression. However, retrovirally derived iPSCs can lead to differentiation failure, as they retrovirally have multiple transgene integrations in the genome, which may result in expression leaks [21]. Another problem is c-Myc (transcription factor), c-Myc can cause tumor formation [22]. Therefore, c-Myc cannot be used as a transcription factor. The significantly reduced when fibroblast-derived iPSCs with only Oct4, Sox2, and KIf4 as a transcription factor. Other methods have been designed to insert reprogramming factors into somatic cells, such as the reduction of integration site by putting the transcription factor, a transposon system encoding a transcription factor, plasmid-based transposon, and expression of reprogramming factor (viral vectors, DNA vectors, and direct protein delivery). The efficiency of iPSCs induction is lower than that with retrovirus vectors. The other transcription factors are used, which are Oct4, Sox2, Nanog, and Lin28. That transcription factor is successfully induced iPSCs. The transcription factor efficiency is enhanced by the addition of extra factors, such as ESRRB, UTF1, Sall4, Tb3, mRNA, and small hairpin RNAs [23].

iPSCs can be generated from MSCs derived from adipose tissue (reprogramming). MSCs derived from diverse iPSCs was the effective source of multipotent cells and have both advantages of iPSCs and traditional MSC, that is greater proliferation capacity in vitro with no time limit, have similar immunomodulatory properties with traditional MSC cells, impairing NK-cells, and have long-term survival after transplantation [12]. While HSC derived from diverse iPSCs have similar characteristics (functional and phenotypic) with human primary HSC and can generate the spheroid cultures that better mimic the complexity of the liver function [13]. iPSCs-MSCs and iPSCs-HSC are safe and effective for cell-based therapy, because of that, iPSCs can be used for various diseases therapy, such as liver fibrosis for thalassemia.

\section{iPSCs-HSCs for Thalassemia Therapy}

HSCs are the key to wound healing in the liver, including liver fibrosis by forming myofibroblasts, increasing ECM production, and reducing degradation. Human primary HSCs are difficult to obtain because of their heterogeneity and limited proliferative capacity. Thus, the HSCs cell line was used, but the cell line didn't have the functional characteristics of the primary HSC. Therefore, an alternative source is needed to obtain HSCs, which is iPSCs. iPSCs have unlimited differentiation ability and potential to differentiate into parenchymal and non-parenchymal liver cells [13].

In the study of Coll et al. 2018 research, $70-80 \%$ of iPSC-HSCs were found, which were characterized by the expression of HSC markers on iPSC-HSCs, there is platelet-derived growth factor receptor- $ß$, vimentin, fibronectin, nerve growth factor, neural cell adhesion molecule, and smooth muscle actin $\alpha$ and protocadherin-7. The method of this research is used several stages of growth factors and compounds (palmitic acid, retinol, fibroblast growth factors, and bone morphogenetic proteins, that play a role during embryonic liver development and obtained the expression of markers, which are the expression of progenitor cells and mesothelial cells during differentiation [13]. The primary function of growth factors and compounds are induced differentiation of ESCs to mesodermal state, induced differentiation of iPSCs to hepatic progenitor cells, and promoted HSCs differentiation (palmitic acid and retinol) [24]. The iPSCsHSCs can promote hepatocyte metabolic function, respond to toxicity, activate and promote intra-spheroid fibrogenesis. As a result, iPSC-HSCs had functional and phenotypic characteristics of human primary cultured HSCs. This method was shown in Figure 1. iPSC-HSCs differ from fibroblasts because iPSCs-HSCs express levels of lecithin retinol acyltransferase which plays a role in the formation of retinyl esters present in lipid droplets to store vitamin C [13].

IPSCs-HSCs have emerged as novel sources of MSCs, HSCs derive from sub-mesothelial cells that showing mesenchymal characteristics. MSCs attenuate liver inflammation by regulating the immune cell function creating a hepato-protective environment through paracrine effect via cell-cell interaction. HSCs also have essential roles in treatment of liver fibrosis by changing their characteristic from quiescent cells to an activated state, producing ECMs [25], [26].

\section{iPSCs Modeling}

\section{Modeling human disease with $2 D$ and $3 D$ culture}

Disease modeling for therapeutic treatment is important for an understanding of human disease pathologies, but that is challenging because of the limited disease modeling. Because of that, iPSCs used for disease modeling with $2 \mathrm{D}$ and $3 \mathrm{D}$ culture because iPSCs are able to differentiate into all body cell types and 
recreate the genetic background of patients. 2D culture systems are the simplest tool than 3D culture systems because using cells plated directly on a rigid substrate supported cell adhesion and cell behavior (proliferation and differentiation). The 2D iPSCs models used to study monogenic and polygenic disease, for example, successful studies for Parkinson's disease. However, 2D iPSC models have to lack information on communication cells, cell-matrix mechanics, the unique in vivo environment, and limitation for application to disease modelings, such as cellular and molecular analysis because 2D iPSCs suitable for modeling early age-onset disease. For improved bioengineering methods, a 3D iPSCs model was used. There are various kinds of $3 D$ iPSCs models, such as engineered tissue, organoid, and organ-on-chip. Engineered tissues successfully used for engineered heart tissue, that is fabricated with hydrogels and supporting cells. Engineered tissue has limited application for cells and inner cell phenotype. Organ on chip used for control tissue composition and architecture in vitro $3 \mathrm{D}$, organ on chip successfully applied for lung, Barth syndrome-associated cardiomyopathy, drug-induced kidney glomerular injury, blood-brain barrier function, and skin wound healing. Organoids are recapitulate the architecture and function of tissue or organ, mostly selforganizing, and exhibit 3D architecture containing many of the cell types found in organs. Organoids iPSCs are successfully used for the brain, liver, gut, lung, kidney, and heart. Furthermore, organoids iPSCs and organ on chip have limited application because they are only at a micrometer to millimeter scale (engineered tissues are centimeter scale). The other lack is iPSC-generated engineering tissues and organoids no supporting tissue, without that, cells cannot access the important nutrients and response signals, so the life span and function of the tissue are limited. The 3D culture system is develop the engineering tissues, but the manufacturing cost is expensive and inconsistent tissue construct quality. Although 2D iPSCs is less adaptable, 2D iPSCs has high reproducibility, greater potential for control, and are acceptable for some application (fetal/early-onset disease) [27]

\section{Bioethical Concerns}

\section{Bioethical concerns about iPSCs}

Stem cells have ethical concerns because the process requires the destruction of embryos. HumaniPSCs (hiPSCs) can be the solution. Derivation hiPSCs from somatic cells does not require the destruction of embryos. However, this study needs to be studied furthermore about the safety of iPSCs therapy, such as abnormal reprogramming, tumorigenicity, cloning humans, producing human germ cells, and making human embryos [28].
Reprograming is needed to induce hiPSCs, but the process is associated with deletions of the tumorsuppressor gene. Generated hiPSCs can be genetically modified; those cause alterations in gene expression in cells and affect differentiation, proliferation, and development. hiPSCs have the ability of unlimited proliferation and able to differentiate into other cell types. If differentiated cells transplanted is uncontrolled, that can cause generate tumor. Cloning humans is used for people who have lost their ability to reproduce and want to have babies. However, human cloning is ethically objectionable. For testing the pluripotency of iPSCs, the tetraploid complementation method is needed. This method is performed by injecting the stem cells into tetraploid embryos, then transferring them to recipients to see the ability of iPSC. But to get the tetraploid embryos, normal diploid embryos are destroyed, and that causes ethical problems. Normal diploid embryos have the potential to form fetuses [28].

After the discovery of the iPSCs, scientists believe that stem cells can be differentiated into germ layers used for artificial reproduction. However, this cause ethical problem, such as artificial germ cells from iPSCs having the same cell morphology and structures as natural germ layers, stability of artificial germ layer without abnormalities of chromosomes and genetic mutation, the ability of fertilization, and their developmental potentiality. Then, to improve the success rate of the research, when a large number of artificial germ cells are produced, only the high-quality embryos are to be selected for transplantation. The poorquality embryos should be destroyed or discarded. The other ethical problem in cloning is iPSCs are genetically modified and then use to obtain offspring with specific characteristics. In practice, that specific characteristic may fail to occur or produce the defective characteristics that are can be carried to next-generation [28].

\section{Biobanking of iPSCs}

Biobanks are an organized collection of biological samples and their related data, which have high quality and safety standards for clinical research. Those biological samples can include cells such as iPSCs. Quality control of the samples is important for biobanks; there are three levels for quality control, which are cell line identity and hygiene, cell performance and stability, and cell product characterization [29].

Short tandem repeat profiling and single nucleotide polymorphism (SNP) genotyping are a method for confirming cell line identity. To identify sample hygiene, microbial analysis is needed. Microbial contamination includes bacterial, fungal, mycoplasma, and virus contamination. The addition of antibiotics to cultured cells can prevent contamination. For detection 
of mycoplasma contamination, direct cultured (broth or agar), and indirect test (indicator culture or DNA dye) can be used [29], [30].

The SNP genotyping is also used for monitoring the genomic stability of cells that can detect small duplication and deletions. The other method for confirming the stability of the cell is karyotyping with Giemsa-banding (G-banding) that used for identification integrity of the chromosome, spectral karyotyping that used for analysis the identification locating translocation, and comparative genome hybridization microarrays that used for identification the integrity of the whole genome [30].

After banking, iPSCs should produce cells representative of the three-germ layer, and after cryopreservation, iPSCs should not be lost their ability to differentiate. The teratomas method is used to test the pluripotency of iPSCs. There is the newest method for analysis of the pluripotency iPSCs and able differentiation, such as gene expression profiles, immunocytochemistry, and flow 4ecytometry staining [30].

\section{Hopes and Challenge}

Research on cell-based therapy with iPSCs continues. Various studies for diseases with iPSCs continue to be carried out. This is because iPSCs have the ability to unlimited self-renewal, differentiate into three germ layers, and can produce unlimited autologous cells for therapy. In addition, iPSCs have also been used as a disease model for heart disease. iPSCs from patients with the long-QT syndrome were used and induced to differentiate into functional cardiac myocytes that recapitulated the electrophysiological defects characteristic of the disorder, but contamination may occur during the growth of iPSCs from adult organs. In addition, it is known that iPSCs can be used to generate functional organs through studies showing that gut and liver from 3D models can be derived into iPSCs. However, the use of iPSCs in clinical application is still being studied further for the safety of iPSCs (tumorigenicity, immunogenicity, and genome instability) [31].

\section{Conclusion}

Cell based therapy with IPSCs-HSCs as a novel sources of MSCs will lead a new option in personalized therapy for thalassemia patients with liver fibrosis mostly due to iron accumulation.

\section{Acknowledgments}

We would like to give special thanks to RSHSUCMSCs therapy for Covid-19 Team, Bandung, for their daily devotions on this pandemic. Also to Universitas Padjadjaran (UNPAD), Bandung, especially Faculty of Medicine, for their technical assistance to publishing this review, Aretha Medika Utama Biomolecular and Biomedical Research Center, Bandung for their valuable assistance to prepare this manuscript.

\section{Availability of Data and Materials Section}

The datasets gathered and shown in this review is available as an open access data as cited in our references; the corresponding author will make it available on reasonable request.

\section{Authors' Contributions}

All authors design the discussion directions, gathering all the data and drafting the manuscript. All authors took part on writing and approved of the final manuscript.

\section{References}

1. Modell B, Darlison M. Global epidemiology of haemoglobin disorders and derived service indicators. Bull World Health Organ. 2008;86(6):480-7. https://doi.org/10.2471/blt.06.036673 PMid: 18568278

2. Wahidiyat, PA. Managing Thalassemia: Insight to Indonesian Practice. Bangkok, Thailand: Paper Presented at: The $30^{\text {th }}$ Regional Congress of the ISBT; 2019. https://doi. org/10.1111/voxs. 12544

3. Khandros E, Weiss MJ. Protein quality control during erythropoiesis and haemoglobin synthesis. Hematol Oncol Clin North Am. 2010;24(6):1071-88. https://doi.org/10.1016/j.hoc.2010.08.013 PMid:21075281

4. Cappellini MD, Farmakis D, Porter J, Taher A, editors. Guidelines for the Management of Transfusion Dependent Thalassaemia (TDT). $4^{\text {th }}$ ed. Nicosia, Cyprus: Thalassaemia International Federation; 2021.

5. Anderson ER, Shah YM. Iron homeostasis in the liver. Compr Physiol. 2013;3(1):315-30. https://doi.org/10.1002/cphy.c120016 PMid:23720289

6. Chaston TB, Matak P, Pourvali K, Srai SK, McKie AT, Sharp PA Hypoxia inhibits hepcidin expression in $\mathrm{HuH} 7$ hepatoma cells via decreased SMAD4 signaling. Cell Physiol. 2011;300(4):C888-95. https://doi.org/10.1152/ajpcell.00121.2010

PMid 21289291 
7. Sikorska K, Bernat A, Wroblewska A. Molecular pathogenesis and clinical consequences of iron overload in liver cirrhosis. Hepatobiliary Pancreat Dis Int. 2016;15(5):461-79. https://doi. org/10.1016/s1499-3872(16)60135-2

\section{PMid:27733315}

8. Philippe MA, Ruddell RG, Ramm GA. Role of iron in hepatic fibrosis: One piece in the puzzle. World J Gastroenterol 2007;13(35):4746-54. https://doi.org/10.3748/wjg.v13.i35.4746 PMid:17729396

9. Elalfy MS, Esmat G, Matter RM, Abdel Aziz HE, Massoud WA Liver fibrosis in young Egyptian beta-thalassemia major patients: Relation to hepatitis $\mathrm{C}$ virus and compliance with chelation. Ann Hepatol. 2013;12(1):54-61. PMid:23293194

10. Mount NM, Ward SJ, Kefalas P, Hyllner J. Cell-based therapy technology classifications and translational challenges. Philos Trans R Soc Lond B Biol Sci. 2015;370(1680):20150017. https://doi.org/10.1098/rstb.2015.0017 PMid:26416686

11. Zakrzewski W, Dobrzyński M, Szymonowicz M, Rybak Z. Stem cells: Past, present, and future. Stem Cell Res Ther. 2019;10(1):1-22. https://doi.org/10.1186/s13287-019-1165-5 PMid:30808416

12. Zomer HD, Vidane AS, Gonçalves NN, Ambrósio CE. Mesenchymal and induced pluripotent stem cells: General insights and clinical perspectives. Stem Cells Cloning. 2015;8:125-34. https://doi.org/10.2147/sccaa.S88036 PMid:26451119

13. Coll M, Perea L, Boon R, Leite SB, Vallverdú J, Mannaerts I, et al. Generation of hepatic stellate cells from human pluripotent stem cells enables in vitro modeling of liver fibrosis. Cell Stem Cell. 2018;23(1):101-13.e7. https://doi.org/10.1016/j. stem.2018.05.027 PMid:30049452

14. Yin C, Evason KJ, Asahina K, Stainier DY. Hepatic stellate cells in liver development, regeneration, and cancer. J Clin Invest. 2013;123(5):1902-10. https://doi.Org/10.1172/JCI66369 PMid:23635788

15. Aydinok Y. Thalassemia. Hematology. 2012;17(1):S28-31. https://doi.org/10.1179/102453312×13336169155295 PMid:22507773

16. Susanah S, Rakhmilla LE, Ghozali M, Trisaputra JO, Moestopo O, Sribudiani $\mathrm{Y}$, et al. Iron status in newly diagnosed $\beta$-thalassemia major: High rate of iron status due to erythropoiesis drive. Biomed Res Int. 2021;26(1):221-4. https:// doi.org/10.1155/2021/5560319 PMid:33954177

17. Motta I, Bou-Fakhredin R, Taher AT, Cappellini MD. Beta thalassemia: New therapeutic options beyond transfusion and iron chelation. Drugs. 2020;80(11):1053-63. https://doi. org/10.1007/s40265-020-01341-9 PMid:32557398

18. Lemischka I. Stem cell dogmas in the genomics era Rev Clin Exp Hematol. 2001;5(1):15-25. https://doi. org/10.1046/j.1468-0734.2001.00030.x PMid:11486729

19. Jiang $\mathrm{N}$, Chen M, Yang G, Xiang L, He L, Hei TK, et al. Hematopoietic stem cells in neural-crest derived bone marrow. Sci Rep. 2016;6(1):36411. https://doi.org/10.1038/srep36411

\section{PMid:28000662}

20. Takahashi K, Yamanaka S. Induction of pluripotent stem cells from mouse embryonic and adult fibroblast cultures by defined factors. Cell. 2006;126(4):663-76. https://doi.org/10.1016/j. cell.2006.07.024

PMid: 16904174

21. Hu K. All roads lead to induced pluripotent stem cells: The technologies of iPSC generation. Stem Cells Dev. 2014;23(12):1285-300. https://doi.org/10.1089/scd.2013.0620 PMid:24524728

22. Hu S, Balakrishnan A, Bok RA, Anderton B, Larson PE, Nelson SJ, et al. 13C-pyruvate imaging reveals alterations in glycolysis that precede c-Myc-induced tumor formation and regression. Cell Metab. 2011;14(1):131-42. https://doi. org/10.1016/j.cmet.2011.04.012

PMid:21723511

23. Chun YS, Byun K, Lee B. Induced pluripotent stem cells and personalized medicine: Current progress and future perspectives. Anat Cell Biol. 2011;44(4):245-55. https://doi. org/10.5115/acb.2011.44.4.245

PMid:22254153

24. Vallverdú J, de la Torre RA, Mannaerts I, Verhulst S, Smout A, Coll M, et al. Directed differentiation of human induced pluripotent stem cells to hepatic stellate cells. Nat Protoc. 2021;16(5):2542-63. https://doi.org/10.1038/ s41596-021-00509-1 PMid:33864055

25. Cao Y, Ji C, Lu L. Mesenchymal stem cell therapy for liver fibrosis/cirrhosis. Ann Transl Med. 2020;8(8):562. https://doi. org/10.21037/atm.2020.02.119

PMid:32775363

26. Koui $Y$, Himeno M, Mori Y, Nakano Y, Saijou E, Tanimizu N, et al. Development of human iPSC-derived quiescent hepatic stellate cell-like cells for drug discovery and in vitro disease modeling. Stem Cell Rep. 2021;16(12):3050-63. https://doi.org/10.1016/j. stemcr.2021.11.002 PMid:34861166

27. Liu C, Oikonomopoulos A, Sayed N, Wu JC. Modeling human diseases with induced pluripotent stem cells: From 2D to $3 D$ and beyond. Development. 2018;145(5):1-6. https://doi.org/10.1242/ dev.156166

PMid:29519889

28. Zheng YL. Some ethical concerns about human induced pluripotent stem cells. Sci Eng Ethics. 2016;22(5):1277-84. https://doi.org/10.1007/s11948-015-9693-6 PMid:26276162

29. Ntai A, Baronchelli S, La Spada A, Moles A, Guffanti A, de Blasio P, et al. A review of research-grade human induced pluripotent stem cells qualification and biobanking processes. Biopreserv Biobank. 2017;15(4):384-92. https://doi.org/10.1089/bio.2016.0097 PMid:28388226

30. Luong MX, Smith KP, Crook JM, Stacey G. Biobanks for pluripotent stem cells. In: Jeanne L, Peterson SE, editors. Human Stem Cell Manual. California, US: Elsevier; 2012.

31. LuX, ZhaoT. Clinical therapy using iPSCs: Hopes and challenges Genomics Proteomics Bioinformatics. 2013;11(5):294-98. https://doi.org/10.1016/j.gpb.2013.09.002 PMid:24060840 